\title{
Analysis of New Demands Promoting the Convergence and Innovation of Data Industry in the Digital Economy
}

\author{
Fei Wang \\ 1Office of Innovation, Entrepreneurship and Employment Guidance Shandong Institute of Commerce and Technology, Jinan, China
}

\begin{abstract}
In the era of digital economy, accelerating the digital transformation of enterprises has become an inevitable trend. Facing new changes and new demands in the new era, the integration and innovation of the data industry is promoted to accelerate the digital transformation of enterprises and to develop informationbased products and services. It can develop targeted products and services on the basis of a clear understanding of user characteristics and needs. Only in this way can it stand on the strategic transformation of the enterprise, promote the integration of the enterprise into the industrial ecological reform, and promote the upgrading of internal management and external marketing. At the same time, this can also make enterprise production management risk controllable through the integration of digital information resources and the application of various digital technologies, and promote the overall upgrading and reform of enterprises. Based on this, this article will put forward some suggestions on how to promote the integration and innovation of the data industry based on the analysis of related concepts, characteristics and new needs in the digital economy era.
\end{abstract}

\section{Overview of the Digital Economy Era}

\subsection{The Meaning of the Digital Economy Era}

Digital economy refers to the combination of digital technology and economy. With the integration of Internet technology and electronic digital technology, new changes have taken place in the digital economy. In the context of the development of data technologies such as cloud computing and big data, digital technology has accelerated the informatization construction of traditional enterprises. It can continuously produce massive amounts of data and new production factors to form new business models. The new concept of digital economy can be used to describe new changes in economic development models. It can use data as the key production factor, network communication technology as the core driving force, and modern information network as the carrier to realize the deep integration and application of various industries and form a new economic development form. The digital economy includes digital industrialization and industrial digitization, which has gone beyond the scope of the industrial sector and has become a technological and economic paradigm and an economic and social form.

\subsection{Characteristics of the Digital Economy Era}

First, in the digital economy era, data has become a new key production factor. Compared with other factors of production, data resources have the characteristics of reproducibility, shareability, and wireless growth and supply, which can break through the limitations of the limited supply of traditional production factors on growth. Secondly, the digital economy provides new impetus for economic innovation. With the wide application of big data, Internet of Things, cloud computing and other technologies, the integrated development of the digital economy has been promoted. The emergence of artificial intelligence, virtual reality, and blockchain has accelerated the improvement of the industrial application ecology, and promoted the acceleration of the digital economy and the integration of manufacturing, biological and energy technologies. In addition, the leading role of the digital technology industry base is very prominent. The rise and innovative development of the digital technology industry have led to the innovative development of other industries. Finally, the biggest driving force in the digital economy era is the integration of industries. The integration of technology and industry has become the main engine of the development of the digital economy. At present, the digital economy continues to expand from consumption to production and from online to offline, giving birth to new business models and new formats, and improving consumption experience and resource utilization.

\subsection{New Changes and New Demands in the Digital Economy Era}

In the era of the digital economy, corporate competition will become more intense. In order to improve their competitive advantage, companies must transform. Business managers must be aware of the importance of adapting to new competition rules to accelerate the digital transformation of enterprises. Second, in the era of digital 
economy, the core of enterprises lies in the research and development of information-based products and services. The information department should quickly enter the new role and turn the IT department into a technical partner with strong business capabilities. In the era of digital economy, the new demand of the data industry is to drive the growth of enterprises with digital revenue. Technology vendors must clearly understand the new needs of users, and lead the company from a technology supplier to a digital transformation partner for users, so as to promote the sustainable development of the company. Besides, as far as the organization is concerned, in order to achieve innovation and development, companies need to actively use new technologies. These technologies include cloud computing, artificial intelligence, VR, etc. These technologies can not only accelerate the transformation of the industry ecology, promote the integration and innovation of the data industry, but also promote the upgrade of corporate marketing, and promote the selfadaptation of security control and risk management.

New KPI

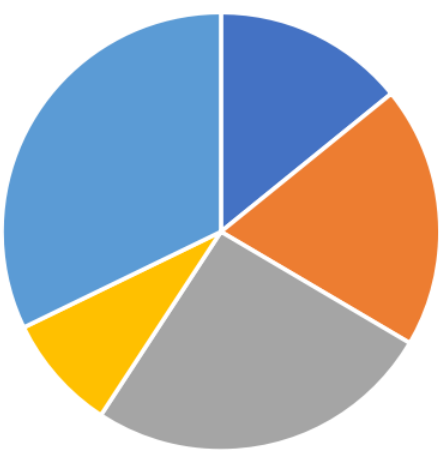

- Digital Guidance - Digital Experience - Digital Information - Digital Operation = Digital Resources

Figure1: Leading the Development of New KPIs for Digital Business

\section{The Strategic Positioning of the Data Industry in the Digital Economy Era}

In the era of big data, the amount of global data is huge. Organizations of all sizes need to deal with large-scale data volumes every day. These data have the characteristics of huge, fast and diverse data. The goal of the data industry is to use data mining and other technologies to dig out information and knowledge with important mechanisms from large-scale data. This information and knowledge can be used in the production, marketing, management and service of the enterprise. The era of the digital economy not only promotes the continuous innovation and development of technologies such as data storage and data analysis, but also promotes accelerated innovation of IT technologies and products, and promotes the vertical integration of the IT industry. Moreover, it also improves the application efficiency of technologies such as data acquisition and data storage, and promotes the capitalization of data. This can transform the information department from cost-centric to profit-centric, promote intelligent decision-making, and promote the transformation of corporate strategy from business-driven to data-driven.

In the digital economy era, the integration and application of a new generation of information technology has become a new focus. Big data will have become the engine of sustained rapid growth in the information industry. Because big data has extremely high commercial value and market demand, it has become a driving force for the continuous rapid development and integration of innovation in the information data industry. Otherwise, corporate decision-making has also shifted from businessdriven to data-driven. We can actively use big data to help companies improve their core competitiveness and quickly seize the market.

\section{Business Architecture of Enterprises in the Digital Economy Era}

\subsection{Industrial Structure}

The structure of the data industry includes data collection and transmission storage, which mainly generate data related to business activities and consumer behavior, and conduct centralized storage of data. Among them, representative companies in the data economy are IBM and HP. Besides, data companies include data storage and data processing that can form valuable information. Representative companies include SAP and Microsoft. In addition, companies that provide data mining and services that can form valuable information and further form knowledge assets include Bloomberg and Google. From the perspective of the development of the data industry, the data economy has gone through the stages of mining industry internal data, integrating internal and external data, user behavior patterns, data mining, and data-driven organizational changes. At present, major domestic and foreign companies have begun to use big data analysis platforms on a large scale. For example, Wal-Mart uses data mining to increase product sales, and the US health department uses drug sales data to predict the epidemic.

\subsection{Business Model}

In the digital economy era, the application of big data technology has given birth to a business model centered on data assets. These models include endogenous value models, exogenous value models, parasitic value models, product value models, and cloud service value models. The endogenous value model refers to the enterprise's analysis of massive data and extraction of valuable information to serve the enterprise's internal production and operation management, and then generate internal economic benefits. The representative industry is the manufacturing industry. The exogenous business model means that companies with big data assets authorize other companies to use the data through lease and sale, thereby turning data assets into income. The representative industries are the telecommunications industry and the financial industry. Parasitic refers to companies with data 
mining and analysis technologies that use big data to provide professional consulting services, but these companies do not produce big data themselves. The product-based value model refers to the ability to provide products related to storage and analysis and obtain income. However, these companies do not generate big data nor rely on big data to provide services and only provide hardware and software products that solve big data problems. For example, Intel Corporation and IBM Corporation. The cloud service value model means that these companies rely on cloud computing to provide users with big data generation, storage, analysis, retrieval, consumption and other service platforms, such as Microsoft cloud platform and Hadoop big data analysis services.

\subsection{Operating Model}

The core of the operating model of each enterprise in the data industry in the digital economy era is the data product model, production model, marketing model and management model. Among them, the iterative update of product models has accelerated, and companies can accurately grasp user needs through data analysis from the source. Afterwards, companies can allow users to participate in product trials, combine user feedback information to improve products, and accelerate the cycle of product upgrades. The intelligent development of production mode means the deep integration of IT technology and manufacturing technology. It achieves personalized customization, which not only improves the efficiency of enterprise operation and reduces costs, but also improves the efficiency of resource utilization. The marketing model is shifting towards precision. This is because the impact environment in the digital economy era is mobile, fragmented, and scene-based. It is no longer limited to consumption at a fixed time and place, but can be consumed through multiple channels around the clock. At this time, consumer subjects have also become personalized, socialized and entertaining, so companies must shift from the product itself to the consumer experience. Companies can use big data marketing to accurately locate users' needs, and then use content marketing, social marketing, and scene-based marketing to improve users' consumption experience. Finally, multiple channels enable companies to directly face users and reduce intermediate costs. From the perspective of enterprise management, in order to solve the problem of internal information asymmetry in the enterprise, the enterprise must actively use the mobile Internet to open up the internal business line of the enterprise. Enterprises also need to strengthen information sharing among enterprises, employees, and customers to achieve synergy in daily business operations. Furthermore, we can use mobile Internet technology to achieve mobile and collaborative business applications, so that employees can handle work anytime, anywhere. This also reduces the cost of collaboration.

\section{Enterprise IT Architecture in the Digital Economy Era}

\subsection{Application Architecture}

Application architecture connects IT and business. Traditional enterprise operation is centered on products, but in the era of digital economy, more attention is paid to user experience. At first, the application architecture of the digital economy needs to expand from focusing on the internal to focusing on the integration of the external and internal and external, and build a user-centric application architecture. The application architecture can first support the management of the enterprise platform ecosystem. That is, enterprise IT construction has developed from single-department construction to multi-department collaborative construction to platform-based ecosystem construction, and the construction of enterprise IT platform realizes the sharing of internal and external resources of the enterprise. Second, the digital economy supports user communities and interaction management, so companies must build their own user communities and strengthen interaction with users. Enterprises must respond to user needs in a timely manner to establish longterm trust relationships. In addition, the digital economy supports online transactions and collaborative management of enterprises. That is to say, enterprises must meet the needs of users to obtain products and services anytime and anywhere, and enterprises can do so by adding third-party trading platforms or self-built platforms Meanwhile, in order to meet the management of human resources, finances, assets, etc., we need to establish a unified large-scale collaboration platform to open up the connections between businesses and realize the exchanges between businesses.

\subsection{Data Architecture}

Currently, companies still have problems in data management, such as different data standards, uneven data quality, and difficulty in data sharing. To this end, companies must establish a clean data architecture to achieve effective data governance. Firstly, in terms of big data collection, the new era enterprise data sources include internal data as well as various Internet data and sensor data. The amount of data is huge and the standards are difficult to unify. To this end, companies need to adopt new data collection technologies, and collect network data with the help of web crawlers and open web APIs. Secondly, companies can use new storage technologies in big data storage and management. For example, Hadoopbased technology can realize data expansion and package storage, and build corresponding databases. In addition, companies also need technologies with higher throughput, fast computing power, and in-depth mining capabilities for data analysis and mining. In terms of big data applications, the most common is visualized data. It can make data information clear and effective through graphical means. 


\subsection{Infrastructure}

In the integration and innovation of the data industry in the digital economy era, only by improving the acumen of the enterprise infrastructure can it not only meet the rapid changes in business, and ensure that infrastructure investment is not increased or reduced. The digital economy has realized the integration of IT platforms through cloud computing technology, and realized the integration of cross-regional and cross-border IT facilities and data resources. Enterprises can quickly obtain relevant resources to meet the needs of business expansion. The use of cloud computing makes enterprises no longer need to invest in IT infrastructure separately.

\section{Corporate IT Governance in the Digital Economy Era}

\subsection{IT Organization Governance}

In the digital economy era, IT technology has been penetrated into a variety of product production, marketing and user services. IT construction is not only to provide technical services to business departments, it is also a business department or a business department partner. Its organizational structure is no longer limited to traditional product development and operation and maintenance, it also includes new functions such as business process optimization and product innovation.

\subsection{IT Project Governance}

From the perspective of project management, data industry companies can use an iterative method for project management. This approach can not only meet the rapidly changing needs of the business, but also improve the effectiveness of project quality control and ensure the smooth completion of the project.

\subsection{IT Operation and Maintenance Governance}

Enterprise IT operation and maintenance is no longer limited to focusing on the internal private cloud of the enterprise, but has begun to focus on the connection between the private cloud and the public cloud. In addition, from the perspective of user experience, enterprises should establish an operation and maintenance model with data as the core. Enterprises can use intelligent and automated technical means to further dig deeper into the value of operation and maintenance data, and provide more accurate decision-making reference basis for daily operation and maintenance to improve the efficiency of operation and maintenance.

\subsection{IT Data Asset Governance}

In the digital economy era, IT resources have broken through organizational boundaries and are developing in the direction of multi-party integration. This can make IT assets, data asset ownership, and usage rights more complicated. As a result, we must establish a more complete data asset governance mechanism, only in this way can we ensure the interests of all parties.

\section{The Development Trend and Development Suggestions of the Data Industry Integration in the Digital Economy Era}

In the era of data economy, data is the core asset. If the data industry first grasps the core asset of data, it can realize the enterprise's market control and obtain huge economic returns. Enterprises can mine more valuable data through the integration and innovation of the data industry. Enterprise-level data warehouse construction will become the mainstream for some time in the future. In the future, the data economy will continue to incorporate multi-source data such as industry data and government public data.

In the era of data economy, the development of the domestic data industry has encountered new problems. Although industrial capital has entered the field of big data, there is still a lack of leading companies that can occupy the big data ecosystem. Although the data is open, the data flow between industries is still insufficient. In consequence, we also need to further strengthen crossindustry integration and innovation. Furthermore, based on the big data ecology, a variety of business value models have been established, and we need to explore more profitable methods.

First of all, in the new era, for the integrated and innovative development of the data industry, enterprises must seize the strategic opportunity of big data and enhance the strategic position of the information industry. The government must formulate active policies and regulations, build a loose environment conducive to the development of big data, improve big data legislation, and ensure the security of data information. Companies need to further observe the new needs of users and focus on innovation in subdivisions. Users need to realize unified and centralized management of data, form enterprise data assets, and dig deeper into the value of big data to promote enterprise intelligent decision-making.

\section{Conclusion}

In summary, data has become an important strategic resource for national enterprises and society. The data economy has continuously become an asset of the organizational structure and a weapon to improve the core competitiveness of an organization. With the innovative development and application of technologies such as the Internet of Things, cloud computing, and data mining, the data economy will drive the rapid development of the data industry chain and open a new era of the IT industry.

\section{References}

1. Lan Qingxin, Dou Kai. The development trend and countermeasures of the digital economy in the sharing 
era [J]. Theoretical Journal, 2017 (6): 8.

2. Zhang Longpeng, Zhang Shuangzhi. Technology empowerment: the technological innovation effect of the integrated development of artificial intelligence and industry [J]. Finance and Economics, 2020 (6): 8.

3. Sun Zhixian. IT industry reform under the digital economy[J]. China Information Technology, 2019 (4): 2.

4. Wen Qiang. Misunderstandings and countermeasures of traditional enterprises' digital transformation [J]. Operation and Management, 2020 (9): 22.

5. Wang Xingshan. Accelerate the digital transformation of enterprises [J]. Construction Enterprise Management, 2017 (12): 14. 